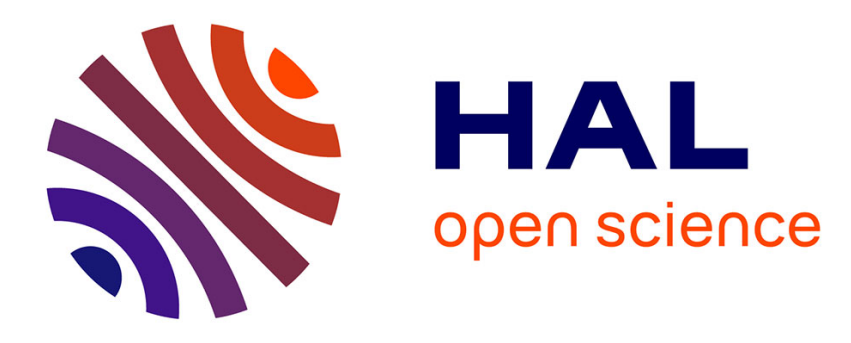

\title{
Efficient Skin Detection Under Severe Illumination Changes and Shadows
}

\author{
Bishesh Khanal, Désiré Sidibé
}

\section{To cite this version:}

Bishesh Khanal, Désiré Sidibé. Efficient Skin Detection Under Severe Illumination Changes and Shadows. ICIRA 2011 - 4th International Conference on Intelligent Robotics and Applications, Dec 2011, Aachen, Germany. pp.1-8. hal-00627903

\section{HAL Id: hal-00627903 \\ https://u-bourgogne.hal.science/hal-00627903}

Submitted on 3 Nov 2011

HAL is a multi-disciplinary open access archive for the deposit and dissemination of scientific research documents, whether they are published or not. The documents may come from teaching and research institutions in France or abroad, or from public or private research centers.
L'archive ouverte pluridisciplinaire HAL, est destinée au dépôt et à la diffusion de documents scientifiques de niveau recherche, publiés ou non, émanant des établissements d'enseignement et de recherche français ou étrangers, des laboratoires publics ou privés. 


\title{
Efficient Skin Detection Under Severe Illumination Changes and Shadows
}

\author{
Bishesh Khanal and Désiré Sidibé \\ Université de Bourgogne, Laboratoire LE2I - UMR CNRS 5158 \\ 12 rue de la fonderie, 71200 Le Creusot, France
}

\begin{abstract}
This paper presents an efficient method for human skin color detection with a mobile platform. The proposed method is based on modeling the skin distribution in a log-chromaticity color space which shows good invariance properties to changing illumination. The method is easy to implement and can cope with the requirements of real-world tasks such as illumination variations, shadows and moving camera. Extensive experiments show the good performance of the proposed method and its robustness against abrupt changes of illumination and shadows.
\end{abstract}

Keywords: Skin detection; Invariance to illumination; Log-chromaticity color space; Face detection

\section{Introduction}

Detecting human skin color is an important step in various applications including video surveillance, visual tracking, content-based image retrieval systems, human computer interaction and robots interaction. In the context of interacting robots, advanced robots should be able to reliably find people in their vicinity and interpret their motion without any a priori knowledge about the environment. Among many visual cues, skin color has proved to be a very useful cue for person detection and for faces or hands detection and tracking.

Color as a low-level feature offers many advantages such as robustness to occlusions, scale variation and geometric transformations. However, the color of an object depends on the illumination conditions, the camera parameters and the reflectance properties of the object. A good skin color detector must be robust against illumination variations and must be able to cope with the great variability of skin color between ethnic groups. Another challenge in detecting human skin color is the fact that many objects in the real world can have skintone colors. For example, wood, leather, skin-colored clothing, hair, etc. This produces a high number of false detections in uncontrolled environment.

Skin color detection can be viewed as a classification problem and the primary step is the selection of a suitable color space, i.e. a color space in which one can easily discriminate between skin and nonskin pixels. Several color spaces have been employed in literature and the choice of the color space affects the performance of any skin detector and its sensitivity to changes in illumination 
conditions $[1,2]$. In most approaches, a color space that de-correlate luminance and chromatic components is adopted, since it is assumed that chromatic components are less sensitive to lighting conditions [3]. However, Cheddad et al. [4] have shown that luminance can be useful in finding skin pixels in color images.

In this paper, we propose the use of a log-chromaticity color space for skin color detection. The log-chromaticity color space shows two important properties. Firstly, a surface color seen under different illuminant colors tends to lie on a straight line in this space. Secondly, for a given camera, the lines corresponding to different surface colors are parallel to each other. Thus an illumination invariant representation of images can be obtained in log-chromaticity color space [5]. We take advantage of this invariant property to develop an efficient skin color detector robust against severe illumination variations and shadows. This robust and fast skin detection algorithm can be used as main input for a module of the software architecture controlling a mobile robot for finding people.

The rest of the paper is organized as follows: previous work on skin detection are described in Section 2, the proposed method is presented in Section 3, experimental and comparative results are shown in Section 4, and conclusions are given in Section 5 .

\section{Related Work}

Human skin color is a very effective feature for persons, faces or hands detection and extensive research has been carried out in developing skin segmentation methods. Most existing techniques involve the classification of image pixels into skin and nonskin categories based on the pixels intensity values. The basic idea behind these techniques is that human skin pixels tend to occupy a small cluster in various color spaces. Therefore, in order to discriminate between skin and nonskin pixels, a color transformation is performed. Skin color detection algorithms are then applied in the new color space.

Many different color spaces are employed in literature including RGB, normalized RGB, HSI, HSV, YCbCr, YES, YUV, CIE Lab [1,2]. Many authors use chrominance color spaces that de-correlate luminance and chromatic components, assuming that chromatic components are less sensitive to lighting conditions than the luminance. In a comparative study of nine chrominance color spaces, Terrillon et al. [3] shows that the tint-saturation-luminance (TSL) space and the normalized RGB space provide best results for Gaussian models. However, it was also shown that discarding the luminance component degrades skin color segmentation results. For example, Cheddad et al. [4] propose a luminance based skin detection method based on deriving an error signal from the grayscale map and the non-red encoded grayscale version of the image. A comprehensive survey of skin color detection in different color spaces can be found in $[1,2]$.

Albiol et al. [6] show that the performance of a skin detection algorithm is largely unaffected by the color space choice, i.e. if an optimal skin detector is designed for every color space, then the performances will be same. Therefore, the classification rules are of most importance. The simplest classification meth- 
ods are classifiers which define cluster boundaries in terms of single or multiple thresholds in the color space. For example, Chai and Ngan [7] propose a facial region segmentation algorithm in the $\mathrm{CbCr}$ plane of the $\mathrm{YCbCr}$ color space. Pixels falling within $C b=[77,127]$ and $C r=[133,173]$ are classified as skin pixels. Sobottka and Pitas [8] propose a set of fixed skin thresholds in the HS plane. The obvious advantage of using explicit thresholding methods is the simplicity of detection rules that leads to a very fast classifier.

Another approach is to use Bayesian classification techniques based on skin color distributions. This family of methods can be divided into parametric and nonparametric methods. Parametric methods assume that skin color distribution follows a known probability density function whose parameters are to be estimated. The parameters are estimated using a training set of skin pixels and a unimodal Gaussian pdf is often adopted [9]. A more sophisticated model, a mixture model, can also be employed [10,3]. However as shown by Caetano et al. [11] the advantage of using a multimodal representation over a unimodal one is limited. The performance of mixture models exceeds single models performance only when a high true positive rate is needed. But a high true positive rate in this case is obtained at the cost of a high false positive rate. In the case of nonparametric methods, the skin color distribution is represented by a lookup table or a histogram. Jones and Rehg [12] use a histogram representation in RGB color space and report better performance in comparison with Gaussian models. Nonparametric methods have the advantage of being independent of the distribution shape. Thus, they can adapt to arbitrary distributions.

A very important aspect of any skin detection algorithm is the robustness against illumination variations since radically changing lighting conditions greatly affect the skin color distribution in an image. Most of the skin detection methods described above can deal only with slight variations in lighting conditions. To achieve robustness against illumination changes, a color constancy algorithm can be used as a preprocessing step. Hsu et al. [9] use the Gray World algorithm to normalize images prior to skin detection while Kakumanu et al. [13] use a neural network based constancy technique. A physics-based approach can also be used to solve the problem of color constancy for skin detection. This approach is based on finding a skin locus, i.e. an area occupied by skin pixels in chromaticity space under certain illuminations. Soriano et al. [14] find the locus directly from images taken under four representative light sources, Horizon (2300K), Incandescent A (2856K), Fluorescent TL84, and daylight $6500 \mathrm{~K}$, and use the obtained locus for skin pixels classification.

\section{Proposed Method}

\subsection{Log-Chromaticity Color Space Properties}

The log-chromaticity color space (LCCS) is a 2D space obtained by taking the logarithm of ratios of color channels. For example, $\log (\mathrm{R} / \mathrm{G})$ and $\log (\mathrm{B} / \mathrm{G})$ form a $2 \mathrm{D}$ LCCS. The illumination invariance property is based on the image formation 
model governed by Eq. 1:

$$
I_{c}=\sigma \int_{\Omega} E(\lambda) S(\lambda) Q_{c}(\lambda) d \lambda, c \in\{R, G, B\} ;
$$

where, $I_{c}$ is the color intensity at a pixel for the color channel $c, \sigma$ is the Lambertian shading, $E$ is the illumination power spectral distribution, $S$ is the surface spectral reflectance function and, $Q_{c}$ is the camera sensor sensitivity function. The integral is computed over the visible spectrum $\Omega$.

Under the assumptions of Planckian lighting, Lambertian surface and a narrowband camera, Finlayson et al. $[5,15]$ shows that the color of a pixel in the LCCS move in a straight line when the illumination is varied. For a given camera, different pixels color in this space are found to be moving in lines parallel to each other, and therefore projecting the pixels in a line perpendicular to these parallel lines, an illumination invariant image can be obtained. To see why this is the case let analyse the implications of the different assumptions.

The narrowband assumption states that the sensor sensitivity functions are exactly Dirac functions $Q_{c}(\lambda)=q_{c} \delta\left(\lambda-\lambda_{c}\right)$ centered at wavelength $\lambda_{c}$. Under this assumption, Eq. 1 becomes:

$$
I_{c}=\sigma E\left(\lambda_{c}\right) S\left(\lambda_{c}\right) q_{c} .
$$

The Planckian assumption implies that lighting can be approximated by Planck's law, in Wien's approximation $[5,16]$ :

$$
E(\lambda, T)=I k_{1} \lambda^{-5} \exp \left(-\frac{k_{2}}{T \lambda}\right),
$$

where $T$ is the illuminant temperature and $k_{1}$ and $k_{2}$ are constants containing the Planck constant, the Boltzman constant and the speed of light in vacuum.

With these assumptions, the color intensity at a pixel is then given by:

$$
I_{c}=\sigma I k_{1} \lambda_{c}^{-5} e^{-\frac{k_{2}}{T \lambda_{c}}} S\left(\lambda_{c}\right) q_{c} .
$$

If, we now form the chromatic components by taking the ratios, for example, of Red and Blue channels w.r.t. Green channel, it is clear from Eq. 4 that intensity and shading information are removed. To remove the nonlinearity, we take the natural logarithm of the ratios and obtain:

$$
\rho_{c}=\log \left(I_{c} / I_{G}\right)=\log \left(s_{c} / s_{G}\right)+\left(e_{c}-e_{G}\right) / T, c \in\{R, B\} ;
$$

with $s_{c}=k_{1} \lambda_{c}^{-5} S\left(\lambda_{c}\right) q_{c}$ and $e_{c}=-k_{2} / \lambda_{c}$.

Eq. 5 shows that in the LCCS all the color values of a surface seen under different illuminants fall on in straigt line. The direction of this line, given by the vector $\left(e_{c}-e_{G}\right)$, is independent of the surface reflectance function. As a consequence, different surface characteristics will produce different lines in the LCCS. However, all these lines are parallel, since they share the same slope (independent from the surface). An invariant image can be formed by projecting the chromatic components into a direction orthogonal to these parallel lines. The result of this projection is a scalar image which is called intrinsic image [5]. 


\subsection{Skin Detection in Log-Chromaticity Color Space}

As stated in Section 2, an important aspect of any skin detection algorithm is its ability to achieve correct classification in the presence of varying illumination conditions. A good skin color detector must be robust against illumination changes and must be able to deal with the great variability of skin color between ethnic groups.

The invariance properties of the log-chromaticity color space can be exploited to develop a robust skin detector. The motivation of our approach is that the skin pixels under different lighting conditions tend to form a distinct cluster in the LCCS. We can thus define a simple classification rule based on the skin cluster boundaries in LCCS. Though other classification techniques such as parametric and nonparametric estimation methods can be used, we have found in our experiments that an explicit thresholding method gives better results (see Section 4.1). Another advantage of using explicit boundaries for classification is the simplicity of detection that leads to a very fast classifier. The boundaries are obtained from a training set of pixels that are manually selected from various images. The images are chosen in order to represent different skin tones, ranging from dark to brownish and whitish, seen under different illumination conditions.

A pixel in an image is classified as a skin pixel if its projection in the LCCS lies in the region defined by: $\log (R / G) \in[0.15 ; 1.1]$ and $\log (B / G) \in[-4 ; 0.3]$. The experiments described in Section 4 show that this simple rule leads to very good detection results in various conditions, providing an efficient solution for human skin color detection.

\section{Experimental Results}

To evaluate the performance of the proposed skin detection method we apply it to a large dataset of images. This dataset used in [17] contains 846 images collected from different existing datasets and is composed of 4.9 million skin pixels and 13.7 million nonskin pixels. We use this dataset for skin detection methods evaluation because it contains ground truth images, i.e. manually segmented skin areas in the images containing humans. With the ground truth images available, it is possible to perform a quantitative analysis of the results. The performances are evaluated in terms of correct detection rate (CDR), false detection rate (FDR) and overall classification rate (CR) [1]. CDR, FDR and CR are, respectively, the percentage of skin pixels correctly classified, the percentage of nonskin pixels incorrectly classified and the percentage of pixels correctly classified.

\subsection{Skin Detection in Log-Chromatic Color Space}

Different classification rules can be employed to detect skin pixels in the LCCS. In this section, we compare a simple direct thresholding method and a parametric method using a Gaussian model (GM). The parameters, mean and covariance, of the GM in LCCS are obtained from the same training set of pixels used to 
find the ranges for the fixed threshold method. Since classification with a GM requires setting a threshold on the probabilities, we vary the threshold from 0 to 1 and find for each value of the threshold the corresponding CDR and FDR. In Table 1, we show the classification performance of the GM in LCCS obtained with different thresholds and the performance of the proposed method with fixed explicit thresholds. As we can see, the fixed thresholds method outperforms the parametric GM approach which, for the same CDR, gives a lot of false positive detection. Moreover, using explicit ranges in LCSS avoid the computation of probabilities or distances, making the detection faster. This justifies our use of explicit threshold ranges for skin detection in LCCS.

Table 1. Comparison of parametric Gaussian model(GM) and explicit thresholds values for skin detection in LCCS.

\begin{tabular}{|c|c|c|c|c|c|c|c|c|c|}
\hline & \multicolumn{8}{|c|}{ GM with varying thresholds $T$} & \multirow{2}{*}{$\begin{array}{l}\text { Proposed method } \\
\text { with fixed threshold }\end{array}$} \\
\hline & $T_{1}$ & $T_{2}$ & $T_{3}$ & $T_{4}$ & $T_{5}$ & $T_{6}$ & $T_{7}$ & $T_{8}$ & \\
\hline CDR & 92.30 & 85.08 & 80.09 & 74.48 & 68.33 & 60.88 & 50.93 & 38.28 & 89.96 \\
\hline FDR & 61.68 & 21.68 & 15.32 & 11.23 & 8.45 & 6.30 & 4.54 & 2.93 & 16.40 \\
\hline
\end{tabular}

\subsection{Comparative Results}

We compared the proposed skin detection method (LCCS) with other popular methods which also use explicit thresholding for classification. The methods considered are the skin locus approach in normalized RGB (NRGB) [18], the use of hue and saturation components in HSV color space (HS) [8], the combination of normalized RGB with hue and saturation (NRGB-HS) [19], the use of chromatic components in $\mathrm{YCbCr}$ color space ( $\mathrm{CbCr})$ [7], and the luminance based method described by Cheddad et al. [4]. For all these methods we use the thresholds given by authors.

Testing on a large dataset showing both indoor and outdoor environments with varying lighting conditions provide an objective comparison of the different methods. Figure 1 shows a few images from the dataset and the detection results using the different methods. The detection results with the entire dataset are summarized in Table 2. We can see that the proposed method compare very well against other opproaches. LCSS leads to an overall classification rate (CR) of about $85 \%$ and is outperformed only by the luminance method which achieves a $\mathrm{CR}$ of $90.45 \%$. On the other hand, the luminance method gives a correct detection rate $(\mathrm{CDR})$ of $68.89 \%$ which much less than $89.96 \%$ given by LCCS. This means that the luminance method misses much more skin pixels than LLCS as can be observed in Figure 1.f and Figure 1.g. The proposed LCCS skin detection method outperforms NRGB, $\mathrm{HS}$ and $\mathrm{CbCr}$ in terms of CDR, FDR and CR. It achieves the best tradeoff between high CDR, detecting as many skin pixels as possible in the images, and low FDR, producing few incorrect detection of nonskin pixels. 

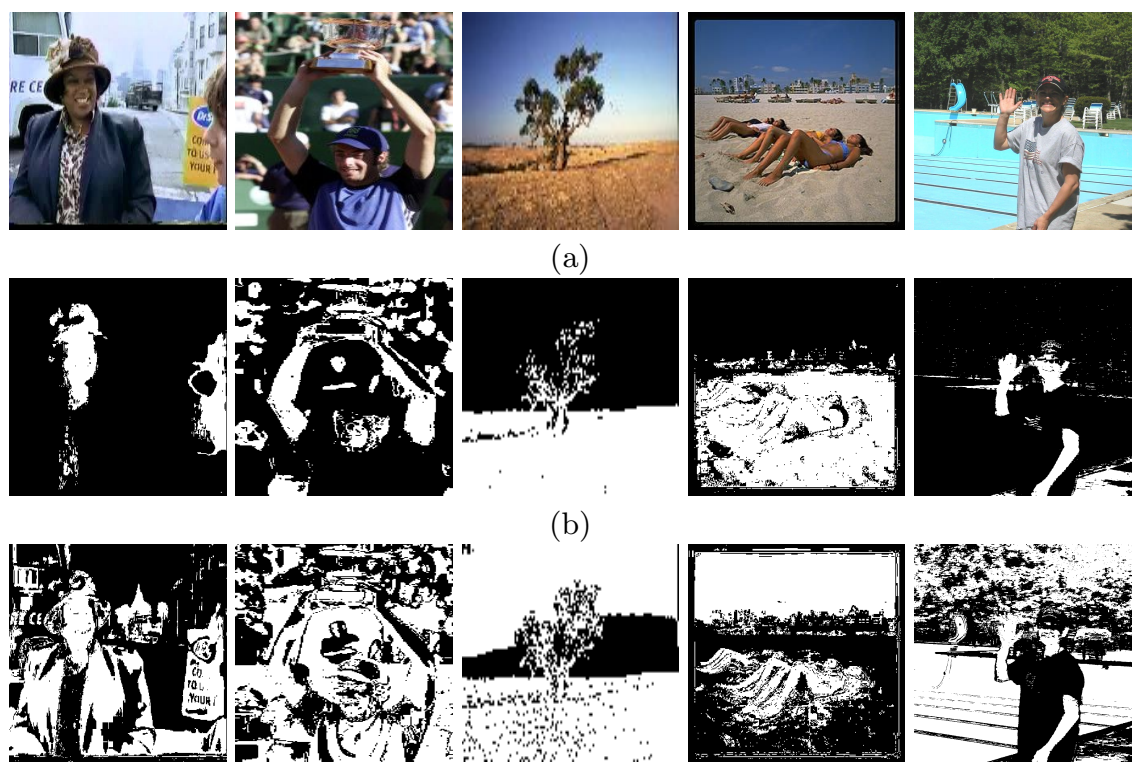

(b)
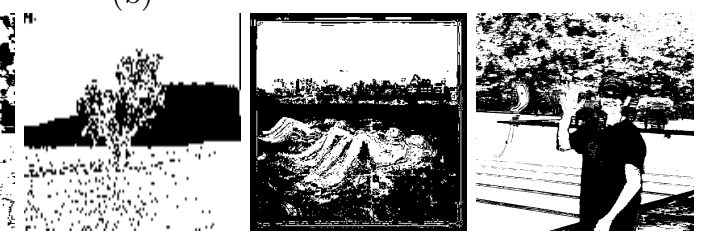

(c)
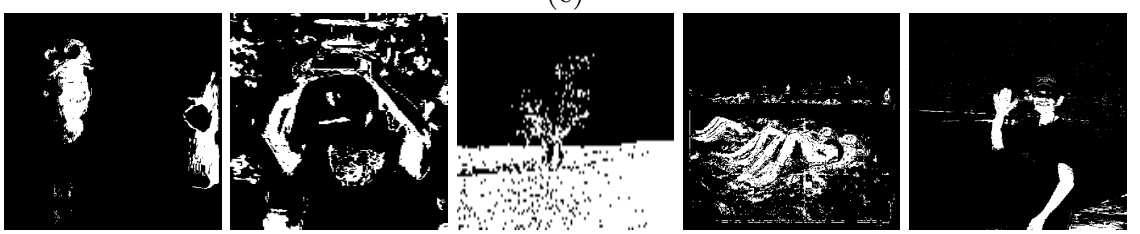

(d)
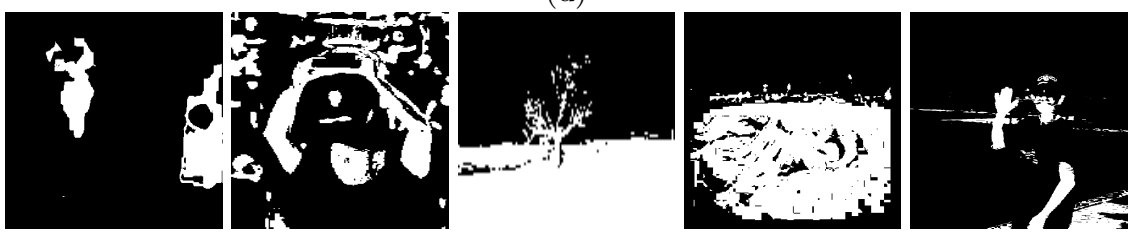

(e)
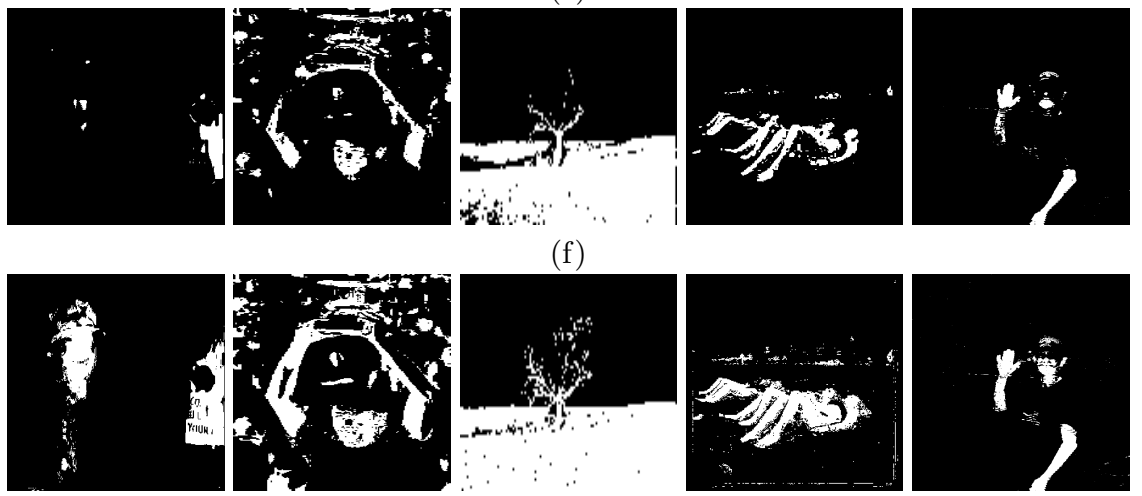

$(\mathrm{g})$

Fig. 1. Some example of skin detection results. (a) Original images. (b) Detection results with NRGB [18]. (c) Detection results with HS [8]. (d) Detection results with NRGB-HS [19]. (e) Detection results with CbCr [7]. (f) Detection results with the luminance method [4]. (g) Detection results with the proposed LCCS method. 
Table 2. Comparison of detection results with the entire dataset.

\begin{tabular}{|c|c|c|c|c|c|c|}
\cline { 2 - 7 } \multicolumn{1}{c|}{} & NRGB [18] & HS [8] & NRGB-HS [19] & CbCr [7] & Luminance [4] & LCCS (proposed) \\
\hline CDR & 70.58 & 63.78 & 47.55 & 89.84 & 68.89 & 89.96 \\
\hline FDR & 17.26 & 29.57 & 11.55 & 22.72 & 5.32 & 16.40 \\
\hline CR & 80.75 & 63.34 & 81.75 & 84.04 & 90.45 & 84.64 \\
\hline
\end{tabular}

\subsection{Robustness Against Illumination Variation and Shadow}

In this section we evaluate the proposed method in the case of severe illumination changes and shadow. This experiment is performed because detecting people with a camera mounted on a mobile robot is one of our future applications. We use a video consisting of three people moving from outdoor bright sunlight to the inside of a building with darker regions. The persons are followed with a handheld camera. The images of the video contain various lighting conditions, from bright sunlight to artificial fluorescent light, and also contains some shadowed areas.

Figure 2 shows some of the results obtained with the video. As can be seen, the proposed method operates well for skin under shadows and abrupt changes in illumination in comparison to other methods. In the third and fourth columns of Figure 2 for example, we can see that the luminance and the $\mathrm{CbCr}$ methods can hardly detect skin pixels and mainly detect the red color of clothing. On the contrary, the proposed LCCS method performs extremely well in such difficult cases. Note how the visible skin areas of the persons are correctly segmented with few false positive detection. Correct classification and false positive rates for this dataset are given in Table 3. The results are obtained using fifty frames taken from the video for which the ground truth images were obtained manually. It contained in total 15.04 million pixels with 0.68 million skin pixels and 14.46 non-skin pixels. As can be seen, the proposed LCCS method outperforms other skin detection methods for this difficult dataset.

Table 3. Detection results with severe illumination changes and shadows.

\begin{tabular}{|c|c|c|c|c|c|c|}
\cline { 2 - 7 } \multicolumn{1}{c|}{} & NRGB [18] & HS [8] & NRGB-HS [19] & CbCr [7] & Luminance [4] & LCCS (proposed) \\
\hline CDR & 35.03 & 41.93 & 22.07 & 40.94 & 29.76 & 64.84 \\
\hline FDR & 4.01 & 32.30 & 1.87 & 6.17 & 4.18 & 4.50 \\
\hline
\end{tabular}

\section{Conclusion}

In this paper an efficient and robust skin detection method is proposed. The method is based on finding human skin locus in the log-chromaticity color space. Based on invariant properties of this color space, a skin detection method is developed and extensive experiments with a large dataset show that the proposed 

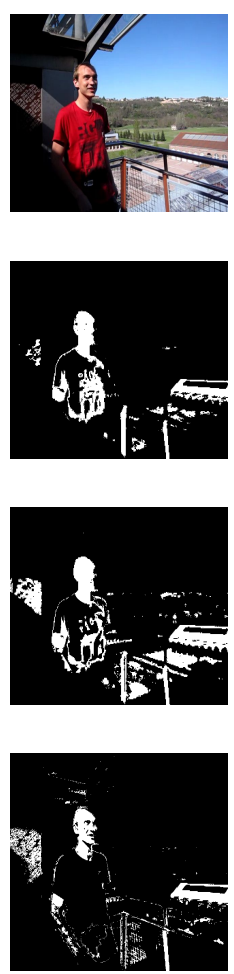
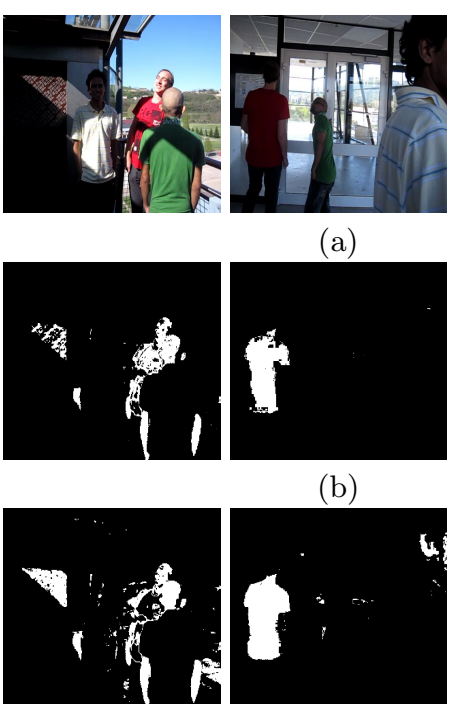

(c)
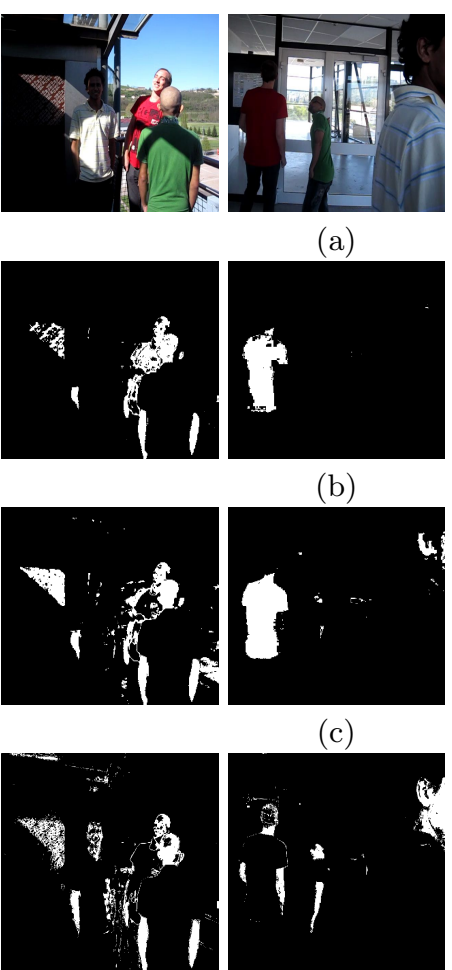

(a)

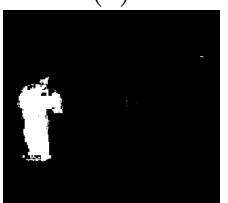

(b)

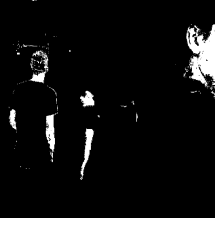

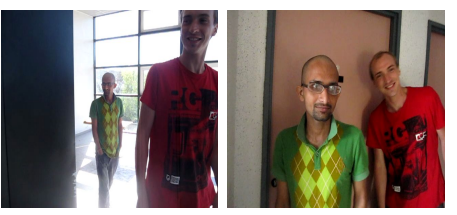
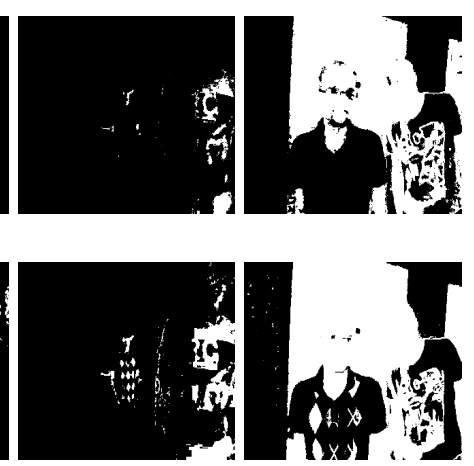

(d)

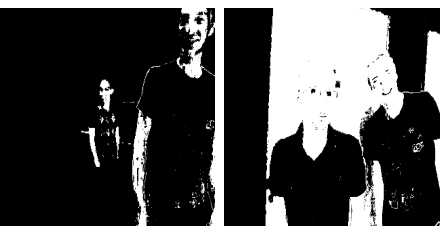

Fig. 2. Testing against illumination and shadow. (a) Original images from the video. (b) Detection results with the luminance method [4]. (c) Detection result with $\mathrm{CbCr}$ [7]. (d) Detection results with LCCS (proposed).

method is particularly robust against severe illumination changes and shadows for images under natural sunlight. Comparison with many other approaches show that the proposed method achieves the best tradeoff between correct classification of skin pixels and false detection of nonskin pixels. The simplicity and the robustness of the method make it suitable for real-world robotic applications. Our future work include using the proposed method for person detection and tracking with a mobile robot.

\section{References}

1. Phung, S. L., Bouzerdoum, A., Chai, D.: Skin segmentation using color pixel classification: analysis and comparison. IEEE Trans. on Pattern Analysis and Machine Intelligence 27(1), 148-154 (2005)

2. Kakumanu, K., Makrogiannis, S., Bourbakis, N.: A survey of skin color modeling and detection methods. Pattern Recognition 40(3), 1106-1122 (2007) 
3. Terrillon, J., Shirazi, M. N., Fukamachi, H., Akamatsu, S.: Comparative performance of different skin chrominance models and chrominance spaces for the automatic detection of human faces in color images. In: IEEE International Conference on Face and Gesture Recognition, pp. 54-61 (2000).

4. Cheddad, A., Condell, J., Curran, V., Mc Kevitt, P., A skin tone detection algorithm for an adaptive approach to steganography. Signal Processing 89(12), 2465-2478 (2009)

5. Finlayson, G., Drew, M., Lu, C.: Intrinsic images by entropy minimization. In: European Conference on Computer Vision. pp. 582-595 (2004)

6. Albiol, A., Torres, L., Delp, E. J.: Optimum color spaces for skin detection. In: IEEE International Conference on Image Processing, pp. 122-124 (2001)

7. Chai, D. Ngan, K. N.: Face segmentation using skin color map in videophone applications. IEEE Trans. on Circuits and Systems for Video Technology 9(4), 551-564 (1999).

8. Sobottka, K., Pitas, I.: A novel method for automatic face segmentation, facial feature extraction and tracking. Signal Processing: Image Communication 12(3), 263-281 (1998).

9. Hsu, R., Abdel-Mottaleb, M., Jain, A. K.: Face detecting in color images. IEEE Trans. on Pattern Analysis and Machine Intelligence 24, 696-706 (2002)

10. Greenspan, H., Goldberger, J., Eshet, I.: Mixture model for face-color modeling and segmentation. Pattern Recognition Letters 22, 1525-1536 (2001)

11. Caetano,T. S., Olabarriaga, S. D., Barone, D. A. C.: Do mixture models in chromaticity space improve skin detection?. Pattern Recognition (36), 3019-3021 (2003)

12. Jones, M., Rehg, J. M.: Statistical color models with application to skin detection. International Journal of Computer Vision 46(1), 81-96 (2002)

13. Kakumanu, P., Makrogiannis, S., Bryll, R., Panchanathan, S., Bourbakis, V.: Image chromatic adaptation using ANNs for skin color adaptation. In: 16th IEEE International Conference on Tools with Artificial Intelligence, pp. 478-485 (2004).

14. Soriano, M., MartinKauppi, J.B., Huovinen, S., Lksonen, M.: Adaptive skin color modeling using the skin locus for selecting training pixels. Pattern Recognition 36 (3), 681690 (2003)

15. Finlayson, G., Drew, M., Lu, C.: Entropy minimization for shadow removal. International Journal of Computer Vision 85, 35-57 (2009)

16. Eibenberger, E., Angelopoulou, E.: The narrow-band assumption in logchromaticity space. In: Color and Reflectance in Imaging and Computer Vision Workshop, in conjunction with ECCV (2010)

17. Schmugge, S.J., Jayaram, S., Shin, M.C., Tsap, L.V.: Objective evaluation of approaches of skin detection using ROC analysis. Computer Vision and Image Understanding 108, 41-51 (2007)

18. Ali, M. R., Morris, T.: Skin locus based skin detection for gesture recognition. In: BMVC UK postgrad. workshop - British Machine Vision Conference pp. 10.1-10.11 (2010).

19. Wang Y., Yuan B.: A novel approach for human face detection from color images under complex background. Pattern Recognition 34 (10) 1983-1992 (2001) 